\title{
Judging age from handwriting done with and without visual feedback
}

\author{
EUGENE A. LOVELACE, BETH A. VELLA, and DONNA M. ANDERSON \\ Alfred University, Alfred, New York
}

\begin{abstract}
In a prior study (Lovelace \& Aikens, 1990), young and old adults wrote 10 common words with their eyes open and again with their eyes closed. The present study used writing samples from 20 old and 20 young writers (67-85 years vs. 18-24 years) in the prior study. Thirty college students judged (1) which were written with eyes closed (paired comparisons, same word written by same person) and (2) whether the writer was young or old. Both tasks were difficult; performance was significantly above chance, but not good ( $71 \%$ correct on open/closed, $65 \%$ on young/old, both $p \mathrm{~s}<.001$ ). Accuracy of open/closed decisions was no higher when judging old writers than young writers, implying that the motor skill of handwriting is well preserved in aged individuals; they do not have an increased need of visual feedback to write. Judges also reported cues they had used to make these decisions. Accuracy of age decisions was significantly related to the reported use of style cues (73\%correct for subjects reporting they used that cue vs. 53\% when not reported, chance $=50 \%$ ).
\end{abstract}

The questions of interest in this study were the following: How well can untrained judges tell the age of a person from a sample of handwriting? Can they judge this more accurately if the person wrote with his/her eyes closed? What sorts of cues might be used to make this judgment?

To provide an initial assessment of answers to these questions, we made use of handwriting samples gathered in another study (Lovelace \& Aikens, 1990). In that study, young and old adults wrote 10 common words of five or six letters (no is or $t \mathrm{~s}$ ) with their eyes open and again with their eyes closed. The present study used writing samples from 20 young adults (18-24 years old) and 20 old adults $(67-85$ years old; $M=73.6, S D=5.2)$ in the prior study. Preliminary analyses of these writing samples, plus the lack of any appreciable age effects on other kinesthetic/motor tasks in the Lovelace and Aikens study, indicated that the motor task of writing might be well preserved in the elderly. The present study allowed a test of discriminability as to whether words were written with eyes open or closed when the same words, written in these two ways by the same individual, were simultaneously present for direct comparison. If the kinesthesis involved in writing, or the accuracy of compiling and executing the motor program to write the words, showed substantial age effects, then the aged would be expected to suffer more when deprived of the opportunity to visually control their writing.

Correspondence should be addressed to E. A. Lovelace, Psychology Division, Alfred University, Alfred, NY 14802.

\section{METHOD}

\section{Subjects}

Thirty students enrolled in introductory psychology courses at Alfred University participated as an option for extra course credit. They ranged in age from 18 to 23 years $(M=19.4, S D=1.3)$.

\section{Procedure}

Words written with eyes closed are often at a slant on the page. To eliminate this cue, words were cut from the page. For each writer, the two versions of each word (written with eyes open vs. closed) were placed side by side in two columns; the left-right position of the eyes-closed version was random, with the restriction that this version occur equally often in the two columns. The 10 words appeared in the same order in all $\mathbf{4 0}$ samples. Order of the samples with respect to age was random.

The students were asked to make two judgments: (1) for each pair of words, they were to decide which was written with eyes closed; and (2) for the entire set of 10 word pairs, they were to decide whether the writer was young or old. After completion of all judgments, the students were asked to describe any cues in the handwriting they were aware of using when making each of the two types of decisions

\section{RESULTS}

\section{Overall Levels of Judgment Accuracy}

Both tasks were viewed by the judges as difficult, but performance was above chance. The open/closed decisions were correct $71 \%$ of the time $(S D=6 \%$, range $52 \%-82 \%)$, which differs significantly from chance $(50 \% ; t=20.5, p<.001)$. Contrary to what one would expect if the aged have reduced kinesthetic-sense ability and so must rely more on visual guidance in writing, there was no difference in the success of these judgments for young $(71 \%)$ versus old $(72 \%)$ writers $(t<1.0)$.

The young/old choices yielded $65 \%$ correct decisions ( $S D=15 \%$, range $33 \%-90 \%)$. Although accuracy of this judgment varied widely, the mean percent correct was 
greater than chance $(t=5.3, p<.001)$. For these decisions, the judges as a group operated in an unbiased manner; half the samples were from young writers, and the mean (and median) proportion "young" responses was .50 (range .38-.65).

\section{Reported Cue Use and Performance}

The cues reported by the students were assigned to categories of similar responses. For the open/closed task, the mean number of cues reported was 3.2 (range 0-6). They fell into the following 10 categories (with $n \mathrm{~s}$ ): spacing (17), neatness (14), size (13), flow/connected (11), letters closed (9), slant (9), spelling (8), uppercase/lowercase (6), pressure (4), and shakiness (4). (Note that since we had cut the words from the page, any remaining slant on the page was largely an artifact of that procedure; thus, no analysis was performed on the slant cue.)

For each of these cues, the data were partitioned on the basis of reported use (or not) of that cue. None of the reported cues was related to performance. The proportion of correct decisions, as a function of reported use versus nonuse of each cue, never differed by more than .05 , and in three cases it actually showed a reversal of direction. None of these differences approached statistical significance. Clearly, there was no substantial relationship between the use of any of the reported cues and accuracy of open/closed decisions.

For the young/old decisions, the mean number of reported cues was 1.7 (range $0-4)$. They fell into the following nine categories with (ns): style (18), neatness (6), size (6), similarity of the open and closed versions (5), spelling (4), shakiness (3), slant (3), spacing (3), and cursive/print (3). Only one cue appeared to be clearly related to performance. The cue that was most widely re-

Table 1

Samples of Reports Categorized as Containing the Style Cue

- Old have letters more evenly distributed; young put more emphasis on first and last letters.

- Old have precise lettering with sort of authority; young didn't have precise lettering.

- Young use "bubble-like" letters, mix in cursive and print; old write more correctly as cursive was taught.

- Old have a fancier style of handwriting; young is more plain.

- Old write in a script that looks experienced; young write bubbly and big.

- Old write more like traditional script; young look straight up and down.

- Old had words more smoothly written, not having rough points and edges.

- Young write in "bubbly letters."

- Old show "penmanship" style of writing.

- Old have more smoothness of flow.

- Old write their letters always the same.

- Old write precise and at an angle-proper letters as supposed to look (as we were taught in 2 nd grade).

- Old write by how they are supposed to be written; young were more "bubble letters."

- Young write up and down, make their own style while old use a traditional style. ported by the subjects, the style cue, was also the cue showing the largest difference in mean proportion of accurate decisions between those reporting they used the cue $(.73)$ and those not reporting its use (.53); recall that the judges were unbiased in this decision; thus, chance is .50 . This was the only cue showing statistical significance associated with its use $(t=4.57, p<.001)$. The differences in proportion-correct decisions based on use/nonuse of other cues ranged between -.03 and .05 , except for the cursive/print cue, which showed a difference of .11 . (This cue partitioned the subjects into groups of 27 nonusers and only 3 users; the $t$ for this difference was significant, 2.72 with $p<.05$, only when separate variance estimates were used).

Descriptions that were coded as including the style category are listed in Table 1.

\section{FOLLOW-UP STUDY}

Could judges who were given the style and cursive/print cues as their criteria make the age decisions with even greater accuracy? Eighteen master's-level students in school psychology at Alfred University were given the same handwriting samples and asked to try to judge the age of each writer using the following criteria: young, bubbly letters, with print and cursive combined; old, traditional script, with precise lettering and letters slanting to right.

This sample showed a slight bias toward saying the writer was old (mean proportion judged "young" $=.43$ ). The overall mean of correct judgments about age was $76 \%$ $(S D=8 \%$, range $=58 \%-88 \%)$, compared with an overall mean correct of $65 \%$ in the above study where no criteria were provided. Although this is a different sample of subjects, so direct comparisons must be regarded with caution, it can be noted that this performance is very similar to the level seen for the subset of subjects in the main study who reported use of these cues $(M=73 \%)$.

\section{CONCLUSIONS}

Judging age from handwriting samples is difficult for most untrained observers (cf. Middleton, 1941); only 65\% correct decisions were made in the two-alternative, forced-choice task of the present study. Certain style cues seem to improve the accuracy of age judgments. Older writers use a more traditional style of cursive penmanship, with letters more precise, smooth and connected, and slanting to the right (cf. Lester, Werling, \& Heinle, 1983). Young adults write in more "bubbly" letters (large rounded forms), and more often mix print and cursive writing.

These style criteria fail to confirm stereotypical notions that the writing of aged individuals will be shaky or disconnected (e.g., Teltscher, 1971) and, instead, suggest that the motor skills and kinesthetic-sense components of this motor task may be well preserved in most healthy older adults (cf. Lovelace \& Aikens, 1990). As Lester et al. (1983) observed about handwriting, "few changes are found with increasing age" (p. 738). The style cues related to increased effectiveness of discrimination in the present study are assumed by the present authors to reflect age (or cohort) differences in penmanship training rather than changes resulting from the aging process.

It is interesting to note that depriving the writers of visual feedback had only small effects for both young and old writers. This parallels 
recent work by Wright (1990). He had college students write with different systems (e.g., their preferred or nonpreferred hands), with and without visual feedback, while writing their name or a "less-wellpracticed" equation. He had anticipated that use of the nonpreferred hand would be disrupted more by the absence of visual feedback, and especially so for the less-well-practiced material, just as Lovelace and Aikens (1990) had predicted that lack of visual feedback would affect the aged more than the young, and more so for the common words than for their name. The following conclusion from Wright's study seems equally applicable to the data of Lovelace and Aikens, which provided the writing samples for the present study: "the data reported here demonstrate that, at the level of overall shape, the trajectories of highly overlearned and less-well-practiced writing movements are similar when performed, with or without visual feedback" (Wright, 1990, p. 315).

More importantly, the absence of visual feedback did not result in any greater alteration of writing for old than for young adults. This implies that for handwriting, neither the compiling or execution of motor programs nor any closed-loop kinesthetic feedback during writing show any appreciable age changes, at least as judged from the overall trajectories (shape) of the written record. It remains to be seen what detailed kinematic analyses might reveal regarding differences between young and old adults in the spatiotemporal changes induced by absence of visual feedback during writing.

\section{REFERENCES}

Lester, D., Werling, N., Heinle, N. H. (1983). Differences in handwriting as a function of age. Perceptual \& Motor Skills, 57, 738.

Lovelace, E. A., Aikens, J. E. (1990). Vision, kinesthesis, and control of hand movement by young and old adults. Perceptual \& Motor Skills, 70, 1131-1137.

MidDleton, W. C. (1941). The ability of untrained subjects to judge intelligence and age from handwriting samples. Journal of Applied Psychology, 25, 331-340.

Teltscher, H. O. (1971). Handwriting: Revelation of self. New York: Hawthorn.

Wright, C. E. (1990). Generalized motor programs: Reexamining claims of effector independence in writing. In M. Jeannerod (Ed.), Attention and performance: Vol. 13. Motor representation and control (pp. 294-320). Hillsdale, NJ: Erlbaum.

(Manuscript received September 10, 1992.) 